\title{
Implicações da pandemia de COVID-19 nos hábitos alimentares de brasileiros:
}

\section{revisão integrativa}

\author{
Implications of the COVID-19 pandemic in brazilian food habits: integrative review \\ Implicaciones de la pandemia del COVID-19 en los hábitos alimentarios brasileños: revisión
}

integrativa

Recebido: 22/03/2021 | Revisado: 30/03/2021 | Aceito: 01/04/2021 | Publicado: 11/04/2021

Evely Rocha Lima

ORCID: https://orcid.org/0000-0002-8989-3313

Universidade Estadual do Sudoeste da Bahia, Brasil E-mail: evely.rl@gmail.com

Thalane Souza Santos Silva

ORCID: https://orcid.org/0000-0002-1732-0137

Universidade Estadual do Sudoeste da Bahia, Brasil

E-mail: thalanesouza@ hotmail.com

Alba Benemérita Alves Vilela

ORCID: https://orcid.org/0000-0003-2110-1751

Universidade Estadual do Sudoeste da Bahia, Brasil

E-mail: abavilela@uesb.edu.br

Vanda Palmarella Rodrigues

ORCID: https://orcid.org/0000-0002-5689-5910 Universidade Estadual do Sudoeste da Bahia, Brasil

E-mail: vprodrigues@uesb.edu.br

Rita Narriman Silva de Oliveira Boery

ORCID: https://orcid.org/0000-0002-7823-9498 Universidade Estadual do Sudoeste da Bahia, Brasil E-mail: rboery@uesb.edu.br

\begin{abstract}
Resumo
A pandemia da COVID-19 alterou a rotina dos brasileiros, gerando impactos diretos nos seus hábitos alimentares. Objetivo: Analisar as implicações da pandemia de COVID-19 nos hábitos alimentares de brasileiros, a partir da produção científica publicada em 2020. Metodologia: Estudo de revisão integrativa da literatura, cuja busca de artigos foi realizada no portal da Biblioteca Virtual em Saúde, em dezembro de 2020, empregando os descritores em inglês: food, eating habits, food consumption, COVID-19, Coronavirus infections. Foram incluídas publicações de artigos originais, de abordagem quantitativa ou qualitativa, realizados no Brasil entre abril e novembro de 2020. Os dados extraídos foram: título, autor, ano, revista, objetivos, metodologia, população/amostra, resultados e conclusões de cada estudo. Resultados: As mudanças alimentares resultantes da pandemia diferiram a depender do grupo etário e das condições socioeconômicas e de saúde do indivíduo. Na maioria dos artigos analisados observou-se impactos negativos, com destaque para o aumento do consumo de alimentos ultraprocessados e redução do consumo de vegetais, especialmente em adultos com depressão, indivíduos de menor renda e escolaridade. O consumo de alimentos não saudáveis frequentemente esteve combinado com o estilo de vida sedentário, representando comportamentos de risco para o desenvolvimento de doenças crônicas não transmissíveis. Entretanto, alguns estudos revelaram mudanças positivas nos hábitos alimentares de adultos, com aumento discreto no consumo de marcadores de alimentação saudável, e redução do consumo de fast foods por adolescentes. Considerações finais: Estudos longitudinais são necessários para melhor compreender os impactos da pandemia da COVID-19 nos hábitos alimentares, bem como para nortear ações de enfrentamento à doença e aos desdobramentos desse fenômeno mundial na saúde dos brasileiros.
\end{abstract}

Palavras-chave: Hábitos alimentares; Consumo alimentar; Pandemia; Infecções por Coronavirus.

\section{Abstract}

The COVID-19 pandemic changed the routine of Brazilians, generating direct impacts on their eating habits. Objective: To analyze the implications of the COVID-19 pandemic on the eating habits of Brazilians, based on the scientific production published in 2020. Methodology: Study of an integrative literature review, whose search for articles was carried out on the Virtual Health Library portal, in December 2020, using the descriptors in English: food, eating habits, food consumption, COVID-19, Coronavirus infections. Publications of original articles, with a quantitative or qualitative approach, carried out in Brazil between April and November 2020 were included. The data extracted were: title, author, year, journal, objectives, methodology, population / sample, results and conclusions of 
each study. Results: The dietary changes resulting from the pandemic differed depending on the age group and the individual's socioeconomic and health conditions. In most of the analyzed articles, negative impacts were observed, with emphasis on the increase in the consumption of ultra-processed foods and reduction in the consumption of vegetables, especially in adults with depression, individuals with lower income and education. The consumption of unhealthy foods has often been combined with a sedentary lifestyle, representing risky behaviors for the development of chronic diseases. However, some studies have shown positive changes in the eating habits of adults, with a slight increase in the consumption of healthy eating markers, and a reduction in the consumption of fast foods by adolescents. Final considerations: Long-term studies are needed to better understand the impacts of the COVID-19 pandemic on eating habits, as well as to guide actions to fight the disease and the consequences of this worldwide phenomenon on the health of brazilians.

Keywords: Eating habits; Food consumption; Pandemic; Coronavirus infections.

\section{Resumen}

La pandemia COVID-19 cambió la rutina de los brasileños, generando impactos directos en sus hábitos alimenticios. Objetivo: Analizar las implicaciones de la pandemia COVID-19 en los hábitos alimentarios de los brasileños, con base en la producción científica publicada en 2020. Metodología: Estudio de una revisión integradora de la literatura, cuya búsqueda de artículos se realizó en el portal Biblioteca Virtual en Salud, en diciembre de 2020, utilizando los descriptores en inglés: alimentos, hábitos alimentarios, consumo de alimentos, COVID-19, infecciones por coronavirus. Se incluyeron publicaciones de artículos originales, con enfoque cuantitativo o cualitativo, realizadas en Brasil entre abril y noviembre de 2020. Los datos extraídos fueron: título, autor, año, revista, objetivos, metodología, población / muestra, resultados y conclusiones de cada estudio. Resultados: Los cambios dietéticos resultantes de la pandemia difirieron según el grupo de edad y las condiciones socioeconómicas y de salud del individuo. En la mayoría de los artículos analizados se observaron impactos negativos, con énfasis en el aumento en el consumo de alimentos ultraprocesados y la reducción en el consumo de verduras, especialmente en adultos con depresión, individuos con menores ingresos y educación. El consumo de alimentos poco saludables a menudo se ha combinado con un estilo de vida sedentario, que representa comportamientos de riesgo para el desarrollo de enfermedades crónicas. Sin embargo, algunos estudios han mostrado cambios positivos en los hábitos alimentarios de los adultos, con un ligero aumento en el consumo de marcadores de alimentación saludable y una reducción en el consumo de comidas rápidas por parte de los adolescentes. Consideraciones finales: Se necesitan estudios a largo plazo para comprender mejor los impactos de la pandemia COVID-19 en los hábitos alimentarios, así como para orientar las acciones para combatir la enfermedad y las consecuencias de este fenómeno mundial en la salud de los brasileños.

Palabras clave: Hábitos alimentarios; Consumo alimentario; Pandemias; Infecciones por Coronavirus.

\section{Introdução}

O ano de 2020 foi marcado pelo surgimento da pandemia causada pelo SARS-CoV-2 (novo coronavírus), notificada em março de 2020, pela Organização Mundial da Saúde - OMS (WHO, 2020a). Diante da gravidade da situação, os países adotaram medidas de contenção da propagação do vírus, o qual apresenta alta capacidade de transmissão (Acter et al., 2020). Práticas comportamentais como, uso de máscaras, frequente higienização das mãos e distanciamento social foram implementadas em todos os países atingidos pela doença, denominada COVID-19, pela OMS (Haushofer \& Metcalf, 2020; WHO, 2020b).

Desde o primeiro caso da doença confirmado no Brasil no mês de fevereiro, os números de diagnósticos confirmados e óbitos cresceram consideravelmente, atingindo quase 7 milhões de casos e mais de 180 mil óbitos até dezembro de 2020 (Rodriguez-Morales et al., 2020; Brasil, 2020). Assim como em todos os países afetados pela doença, o Brasil também adotou medidas de distanciamento social, suspendendo o funcionamento de órgãos públicos, comércio, restaurantes, bares, igrejas, além de escolas e universidades (Menezes Morato et al., 2020).

Neste contexto, os brasileiros foram obrigados a mudar suas rotinas, alterando seu local de trabalho para o home office, muitos com seus filhos em ensino on-line, além daqueles que perderam seus empregos diante da crise econômica desencadeada pela pandemia no país (Gomes, 2020; Klering \& Neto, 2020; Veiga et al., 2020). Considerando esse cenário, os impactos causados pela pandemia da COVID-19 na saúde mental podem ser observados, seja por medo de ser contaminado pelo vírus ou pelas mudanças ocasionadas pela pandemia (Brooks et al., 2020; Ornell et al., 2020).

Sintomas relacionados à depressão, ansiedade, preocupação, estresse e solidão durante a pandemia da COVID-19 
foram evidenciados por Pan et al. (2020) e Sayeed et al. (2020) com holandeses e universitários de Bangladesh, respectivamente. Vale destacar que o estado de saúde mental pode influenciar diretamente na alimentação, pois de acordo com Mantau et al. (2018), pessoas tendem a regular o humor negativo por meio do consumo de alimentos não saudáveis, fenômeno chamado de "alimentação emocional".

Segundo Mattioli (2020), os impactos na saúde mental ocasionados pelo distanciamento social na pandemia, como ansiedade e estresse estão associados a um estilo de vida pouco saudável, contribuindo para o desenvolvimento de Doenças Crônicas Não Transmissíveis (DCNT). López-Moreno et al. (2020) relataram aumento de peso, não realização de atividades físicas, prejuízos no sono e prática da alimentação emocional pela maioria dos participantes do estudo realizado com a população espanhola durante a pandemia.

Diante disso, pode-se esperar um maior consumo de alimentos processados, aumento na demanda por serviços delivery de alimentos e, em alguns casos, mais tempo para se dedicar às práticas culinárias, produzindo alimentos ricos em açúcares e gorduras trans, o que contribui diretamente para o desenvolvimento das DCNT (Marrón-Ponce et al., 2019). Vale ressaltar que estudos revelam a relação direta das DCNT, como obesidade, diabetes mellitus tipo II e hipertensão arterial sistêmica no agravamento da COVID-19, podendo resultar em síndrome respiratória aguda, evoluir para pneumonia viral grave e, até mesmo, falência múltipla de órgãos, levando ao óbito (Lur et al., 2020; Sosa et al., 2020).

Neste sentido, este estudo tem como objetivo, analisar as implicações da pandemia da COVID-19 nos hábitos alimentares dos brasileiros, a partir da produção científica publicada em 2020.

\section{Metodologia}

Trata-se de uma revisão integrativa da literatura, que conta com método sistematizado para síntese e análise da literatura científica disponível. A revisão seguiu os passos metodológicos propostos por Souza et al. (2010): 1) definição da questão norteadora; 2) elaboração da estratégia e execução da busca; 3) seleção dos estudos encontrados; 4) escolha das informações relevantes nos estudos selecionados; 4) análise crítica dos estudos incluídos; 5) discussão dos resultados; 6) apresentação da revisão integrativa.

A questão norteadora foi definida como: "quais as implicações da pandemia de COVID-19 nos hábitos alimentares de brasileiros?”. A busca foi realizada no mês de dezembro de 2020 no portal da Biblioteca Virtual em Saúde (BVS), utilizando os descritores em inglês: food; eating habit; food consumption; COVID-19; Coronavirus infections; combinados pelo operador booleano AND.

Foram considerados elegíveis artigos originais, de abordagem quantitativa ou qualitativa, realizados no Brasil, entre abril e novembro de 2020, disponíveis em texto completo de acesso gratuito, nos idiomas inglês, português ou espanhol e que contemplassem a questão norteadora proposta.

O processo de seleção dos artigos, realizado por dois pesquisadores conjuntamente, iniciou-se pela leitura dos títulos e resumos, que permitiu a exclusão de duplicatas e de trabalhos que não se enquadravam na temática ou nos critérios de elegibilidade. Seguiu-se com a leitura na íntegra dos artigos restantes e todos foram considerados elegíveis para inclusão no estudo.

Os dados extraídos dos artigos selecionados incluíram: título, autor, ano, revista, objetivos, metodologia, população/amostra, resultados e conclusões de cada estudo, sendo que os principais dados foram sistematizados e apresentados em forma de quadro na seção dos resultados. 


\section{Resultados e Discussão}

Dos 56 artigos encontrados nas bases de dados, foram incluídos sete estudos que atendiam aos critérios de inclusão e abordavam a questão norteadora (Figura 1). A caracterização dos estudos é apresentada no Quadro 1.

Figura 1. Fluxograma de seleção dos artigos incluídos no estudo.

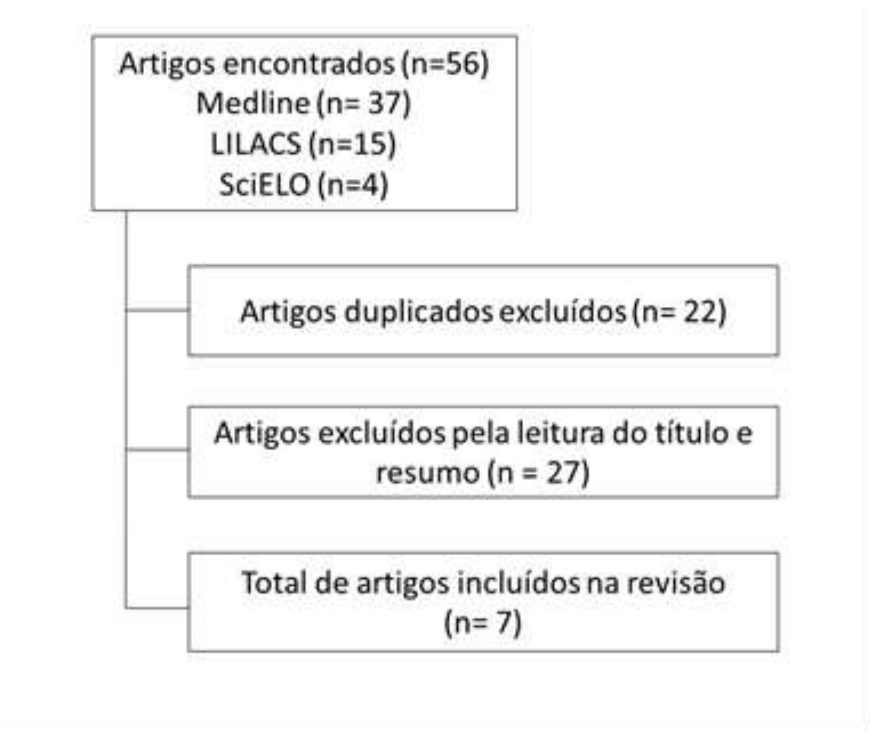

Fonte: Autores (2021).

A maior parte dos artigos ( $\mathrm{n}=5)$ refere-se a estudos com adultos (Malta et al., 2020; Steele et al., 2020; Werneck et al., 2020a), adultos com e sem depressão (Werneck et al., 2020b) e com pais de crianças entre 0 e 12 anos (Campagnaro et al., 2020). Um estudo envolveu uma amostra específica de pacientes pós-bariátricos de São Paulo (Nicoletti et al., 2020) e outro avaliou adolescentes de quatro países, incluindo o Brasil (Ruiz-Roso et al., 2020). Quanto a metodologia, todas as pesquisas seguiram o delineamento observacional, sendo um estudo de coorte (Steele et al., 2020) e dois estudos (Werneck et al., 2020ab) transversais do tipo antes e depois da quarentena.

A utilização de questionários via web para coleta de dados foi verificada em seis artigos. Em apenas um, a coleta ocorreu por inquérito telefônico (Nicoletti et al., 2020). De fato, a coleta de dados mediada por tecnologias tem sido frequente nos estudos em saúde no último ano, em decorrência das restrições impostas pela pandemia. Apesar das dificuldades e dos possíveis vieses, especialmente em se tratando da investigação sobre consumo alimentar, essa modalidade tem sido bem aceita e replicada por pesquisadores de todo o mundo.

Em relação aos objetivos, apenas dois trabalhos avaliaram exclusivamente as mudanças alimentares no contexto da pandemia (Steele et al., 2020; Nicoletti et al., 2020). Nos outros artigos os autores investigaram também variáveis como o comportamento sedentário, tempo em equipamentos eletrônicos com tela, uso de tabaco e álcool, medo e percepções sobre saúde bucal. Destaca-se em todos os estudos a utilização da classificação dos alimentos quanto ao nível de processamento na avaliação da alimentação de forma geral ou na idealização de marcadores de alimentação saudável e não saudável. 
Quadro 1. Caracterização dos estudos publicados sobre hábitos alimentares durante a pandemia da COVID-19 no Brasil.

\begin{tabular}{|c|c|c|c|c|}
\hline Título/Periódico & Autoria & Objetivos & Método & $\begin{array}{l}\text { Conclusões/ Implicações da } \\
\text { COVID-19 }\end{array}$ \\
\hline $\begin{array}{l}\text { COVID-19 pandemic and } \\
\text { pediatric dentistry: fear, } \\
\text { eating habits and parent's } \\
\text { oral health } \\
\text { perceptions/Children and } \\
\text { Youth Services Review }\end{array}$ & $\begin{array}{l}\text { Campagnaro } \\
\text { et al. }\end{array}$ & $\begin{array}{l}\text { Avaliar o impacto da pandemia } \\
\text { sobre o medo, as escolhas } \\
\text { alimentares e as percepções dos } \\
\text { pais sobre a saúde bucal. }\end{array}$ & $\begin{array}{l}\text { Estudo transversal realizado entre } \\
\text { maio a junho de } 2020 \text { com coleta } \\
\text { realizada através de formulário } \\
\text { on-line, que abordavam sobre } \\
\text { dados sociodemográficos, hábitos } \\
\text { alimentares e percepção da saúde } \\
\text { bucal dos pais das crianças. }\end{array}$ & $\begin{array}{l}\text { A maioria das famílias apresentou } \\
\text { mudanças na rotina diária e nos } \\
\text { hábitos alimentares durante a } \\
\text { pandemia da COVID-19, sendo } \\
\text { observada uma associação entre a } \\
\text { redução de renda e aumento no } \\
\text { consumo de alimentos mais } \\
\text { baratos, bem como de alimentos } \\
\text { processados. }\end{array}$ \\
\hline $\begin{array}{l}\text { The COVID-19 Pandemic } \\
\text { and the changes in the } \\
\text { lifestyle of adult } \\
\text { Brazilians: a r cross- } \\
\text { sectional } \\
\text { 2020/Epidemiologia } \\
\text { Serviços de Saúde }\end{array}$ & Malta et al. & $\begin{array}{l}\text { Descrever as mudanças no } \\
\text { estilo de vida, quanto ao } \\
\text { consumo de tabaco, bebidas } \\
\text { alcoólicas, alimentação e } \\
\text { atividade física, no período de } \\
\text { restrição social consequente à } \\
\text { pandemia da COVID-19. }\end{array}$ & $\begin{array}{l}\text { Estudo transversal realizado de } \\
\text { abril a maio de } 2020 \text { com coleta } \\
\text { realizada através de formulário } \\
\text { on-line, que abordava sobre o } \\
\text { consumo de cigarros, bebida } \\
\text { alcoólica, alimentos e prática de } \\
\text { atividade física, bem como uso de } \\
\text { equipamentos eletrônicos com } \\
\text { tela. }\end{array}$ & $\begin{array}{l}\text { Durante a pandemia foi } \\
\text { observado aumento no consumo } \\
\text { de tabaco e álcool, bem como de } \\
\text { alimentos processados e redução } \\
\text { de prática de atividades físicas e } \\
\text { maior tempo dedicado a } \\
\text { atividades sedentárias. }\end{array}$ \\
\hline $\begin{array}{l}\text { Nutritional Inadequacies } \\
\text { Among Post-bariatric } \\
\text { Patients During COVID-19 } \\
\text { Quarantine in Sao Paulo, } \\
\text { Brazil/ Obesity Surgery }\end{array}$ & $\begin{array}{l}\text { Nicoletti et } \\
\text { al. }\end{array}$ & $\begin{array}{l}\text { Investigar os hábitos } \\
\text { alimentares e ingestão de } \\
\text { alimentos durante a quarentena } \\
\text { de COVID-19 em pacientes } \\
\text { submetidos recentemente a } \\
\text { cirurgia bariátrica. }\end{array}$ & $\begin{array}{l}\text { Estudo observacional com coleta } \\
\text { realizada através de inquérito } \\
\text { telefônico, que investigou o } \\
\text { consumo de alimentos durante a } \\
\text { quarentena por meio de } \\
\text { recordatórios alimentares de } 24 \\
\text { horas. }\end{array}$ & $\begin{array}{l}\text { Foi observado baixo consumo de } \\
\text { proteínas e uma ingestão } \\
\text { relativamente alta de alimentos } \\
\text { ultraprocessados. }\end{array}$ \\
\hline $\begin{array}{l}\text { Covid-19 Confinement and } \\
\text { Changes of Adolescent's } \\
\text { Dietary Trends in Italy, } \\
\text { Spain, Chile, Colombia } \\
\text { and Brazil/ Nutrients }\end{array}$ & $\begin{array}{l}\text { Ruiz-Roso et } \\
\text { al. }\end{array}$ & $\begin{array}{l}\text { Estudar as modificações } \\
\text { nutricionais durante o } \\
\text { confinamento por COVID-19 } \\
\text { em adolescentes, comparando- } \\
\text { os com sua dieta habitual e } \\
\text { diretrizes e identificar as } \\
\text { variáveis que podem ter } \\
\text { influenciado as mudanças. }\end{array}$ & $\begin{array}{l}\text { Estudo transversal realizado de } \\
\text { abril e maio de } 2020 \text { com a coleta } \\
\text { realizada por meio de formulário } \\
\text { on-line, que abordava sobre } \\
\text { hábitos alimentares. }\end{array}$ & $\begin{array}{l}\text { Foi observado aumento do } \\
\text { consumo de frutas e vegetais e } \\
\text { redução no consumo de fast-foods } \\
\text { por adolescentes brasileiros } \\
\text { durante a pandemia. Entretanto, } \\
\text { observou-se aumento do consumo } \\
\text { de alimentos doces e bebidas } \\
\text { açucaradas. }\end{array}$ \\
\hline $\begin{array}{l}\text { Associations of sedentary } \\
\text { behaviours and incidence } \\
\text { of unhealthy diet during } \\
\text { the COVID-19 quarantine } \\
\text { in Brazil/Public Health } \\
\text { Nutrition }\end{array}$ & $\begin{array}{l}\text { Werneck et } \\
\text { al. }\end{array}$ & $\begin{array}{l}\text { Analisar a associação de } \\
\text { padrões de mudança em assistir } \\
\text { TV e uso de computador/tablet } \\
\text { e incidência de consumo } \\
\text { elevado de consumo de } \\
\text { alimentos ultraprocessados e } \\
\text { menor consumo de frutas e } \\
\text { vegetais durante a pandemia } \\
\text { COVID-19. }\end{array}$ & $\begin{array}{l}\text { Estudo transversal realizado de } \\
\text { abril a maio de } 2020 \text { com dados } \\
\text { retrospectivos coletados por } \\
\text { questionário via web. Foram } \\
\text { avaliados hábitos alimentares } \\
\text { saudáveis e não saudáveis, bem } \\
\text { como o tempo de uso de } \\
\text { TV/computador e tablets. }\end{array}$ & $\begin{array}{l}\text { Participantes com incidência de } \\
\text { comportamentos sedentários } \\
\text { foram mais propensos a } \\
\text { apresentar frequência elevada de } \\
\text { consumo de alimentos } \\
\text { ultraprocessados e baixo consumo } \\
\text { de frutas e vegetais durante a } \\
\text { quarentena de COVID-19. }\end{array}$ \\
\hline \begin{tabular}{lrr} 
Lifestyle & \multicolumn{2}{c}{ behaviors } \\
changes & during & the \\
COVID-19 & pandemic \\
quarantine & among & 6,881 \\
Brazilian & adults & with \\
depression & and & 35,143 \\
without depression/Ciência \\
e saúde coletiva
\end{tabular} & $\begin{array}{l}\text { Werneck et } \\
\text { al. }\end{array}$ & $\begin{array}{l}\text { Analisar a associação entre } \\
\text { depressão previamente } \\
\text { diagnosticada e alterações na } \\
\text { atividade física, tempo } \\
\text { assistindo TV, consumo de } \\
\text { frutas e vegetais, bem como na } \\
\text { frequência do consumo de } \\
\text { alimentos ultraprocessados. }\end{array}$ & $\begin{array}{l}\text { Estudo transversal realizado de } \\
\text { abril a maio de } 2020 \text { com dados } \\
\text { retrospectivos coletados por } \\
\text { questionário via web. Foram } \\
\text { avaliados comportamentos de } \\
\text { risco relacionados à alimentação, } \\
\text { prática de atividade física e tempo } \\
\text { assistindo TV. }\end{array}$ & $\begin{array}{l}\text { Os participantes com diagnóstico } \\
\text { prévio de depressão estavam em } \\
\text { risco de incidência de } \\
\text { comportamentos alimentares não } \\
\text { saudáveis durante a quarentena. }\end{array}$ \\
\hline
\end{tabular}


Quanto aos resultados, de forma geral, verificou-se mudanças desfavoráveis relacionadas aos hábitos alimentares dos brasileiros durante a pandemia. Em comparação ao período que antecedeu a quarentena houve aumento do consumo de alimentos ultraprocessados e redução da ingestão de frutas e vegetais (Malta et al., 2020; Werneck et al., 2020ab). Da mesma forma, no estudo realizado durante a pandemia da COVID-19 com universitários brasileiros, da Mota Santana et al. (2021) observaram alterações no peso e na dieta dos participantes. Os autores destacaram que o aumento do peso se associou ao consumo de alimentos processados e ultraprocessados, revelando a implicação negativa do distanciamento social no consumo alimentar dos universitários, ao considerar os impactos na saúde em virtude do excesso de peso que pode levar à obesidade.

Segundo Menegassi et al. (2018), a classificação dos alimentos baseada no seu nível de processamento se fundamenta a partir do avanço no desenvolvimento industrial de alimentos, bem como nas demandas contemporâneas socioeconômicas da sociedade. Neste sentido, ao longo dos anos houve aumento no consumo de alimentos ultraprocessados, como observado na coorte realizada por Bielemann et al. (2015) com adultos jovens, que revelou um alto consumo desses alimentos e uma associação positiva com o consumo de sódio, colesterol e gorduras.

É importante salientar que o consumo de alimentos ultraprocessados está intimamente relacionado a um estilo de vida não saudável, sedentarismo e desenvolvimento das DCNT (Who, 2003). Segundo o estudo de coorte realizado na Espanha por Blanco-Rojo et al. (2019), o consumo de alimentos ultraprocessados aumentou o risco de mortalidade na população em geral, enquanto a substituição dessa classe de alimentos por aqueles não processados ou minimamente processados poderia reduzir este risco. Complementarmente, o Global Burden of Disease Study (2018), evidenciou que as DCNT se apresentam como a maior fração de causa de mortes em todo o mundo, revelando a necessidade de hábitos que reduzam o risco de adquirir tais doenças como, por exemplo, hábitos alimentares mais saudáveis.

Destaca-se que os hábitos alimentares podem ser influenciados por uma série de fatores, como a saúde física e mental. Werneck et al. (2020b) observaram que pacientes com depressão apresentaram maior chance de incidência de frequência elevada de consumo de alimentos ultraprocessados comparando-se com pessoas sem depressão. Da mesma forma, McAtamney et al. (2021), avaliando o impacto da pandemia no comportamento alimentar de indivíduos no Reino Unido, observaram aumento no consumo de alimentos não saudáveis, bem como o relato de comerem mais do que o habitual durante a pandemia. Os autores verificaram ainda que, os participantes que relataram alterações no comportamento alimentar, também relataram aumento nos sintomas relacionados à depressão durante a pandemia, sugerindo a prática da alimentação emocional.

Seguindo nessa problemática, no estudo realizado com italianos por Cecchetto et al. (2021), foi evidenciado um aumento da alimentação emocional associada à maior depressão, ansiedade, qualidade das relações pessoais e qualidade de vida, além do aumento da compulsão alimentar ocasionado por maior estresse durante a pandemia da COVID-19.

Ainda entre os estudos avaliados, o de coorte prospectiva conduzido por Steele et al. (2020) verificou aumento discreto, porém estatisticamente significante, no consumo de marcadores de alimentação saudável e estabilidade no consumo de marcadores de alimentação não saudável durante a pandemia, o que representa um resultado positivo. Tais resultados podem estar relacionados ao aumento da preocupação das pessoas com a saúde em tempos de pandemia, uma vez que se sabe que uma alimentação saudável pode contribuir para o fortalecimento do sistema imunológico (Dias et al., 2020). Além disso, é possível considerar que o fato de as pessoas estarem mais tempo em casa, em decorrência do distanciamento social, pode contribuir para a prática da alimentação em família. Estudos realizados por Suggs et al. (2018) e Jones (2018) revelam que o hábito de realizar refeições em família pode contribuir para a prática de uma alimentação mais saudável.

Steele et al. (2020) observaram também aumento no consumo de alimentos ultraprocessados nas regiões economicamente menos desenvolvidas e por pessoas com menor escolaridade, sugerindo que desigualdades socioeconômicas possam influenciar na resposta à pandemia. Segundo Torres et al. (2020), o poder aquisitivo pode influenciar diretamente nos hábitos alimentares, uma vez que o custo dos alimentos pode ser determinante no processo de escolha alimentar. De acordo 
com o estudo realizado por Penne e Goedemé (2020), fatores como renda familiar e políticas de renda mínima podem limitar o acesso regular a uma alimentação saudável e variada. Dessa forma, indivíduos com menor poder aquisitivo tendem a optar por alimentos ricos em açúcares, gorduras e sódio, frequentemente mais acessíveis e com maior rendimento, porém fortemente associados ao desenvolvimento da obesidade e DCNT.

Alterações positivas nos hábitos alimentares também foram observadas no estudo de Ruiz-Roso et al. (2020), que verificaram aumento do consumo de vegetais e redução do consumo semanal de fast-foods, considerando a subamostra de adolescentes brasileiros incluídos. Os autores atribuem esses resultados a maior disponibilidade de tempo das famílias para a preparação de refeições no domicílio. Segundo Mills et al. (2017), a preparação de comida em casa compõe o conjunto de medidas de combate a obesidade e a dietas de baixa qualidade, condições bastante prevalentes entre os adolescentes. Entretanto, o confinamento ocasionou também tédio e estresse, o que pode justificar o aumento da ingestão de alimentos doces e bebidas açucaradas observado entre os jovens brasileiros, trazendo novamente ao debate o conceito da alimentação emocional (Cecchetto et al., 2021).

Ao serem comparados aos adolescentes italianos, espanhóis, chilenos e colombianos, o estudo revelou um maior consumo de legumes pelos brasileiros, bem como aumento no consumo desse grupo alimentar durante a pandemia da COVID19 em relação ao período anterior (Ruiz-Roso et al., 2020). Este fato, pode refletir a influência do país de residência no consumo alimentar, considerando os fatores socioeconômicos, bem como aspectos culturais, religiosos e tradições de cada nação (Zarnowiecki et al., 2014).

No estudo de Campagnaro et al. (2020), 61,5\% dos adultos investigados revelaram mudanças no padrão alimentar dos seus lares, destes, $33,1 \%$ disseram que estavam escolhendo alimentos mais saudáveis, enquanto os demais relataram aumento no consumo de alimentos processados, massas, alimentos congelados e salgadinhos. Do ponto de vista da condição econômica, as famílias com perda significativa de renda relataram estar comendo menos do que antes ou optando por alimentos de menor custo.

Neste sentido, observa-se a implicação da pandemia da COVID-19, bem como das medidas de distanciamento social nos aspectos socioeconômicos, representado pela redução de renda das famílias, fato que pode contribuir para o aumento da insegurança alimentar e nutricional (Ribeiro-Silva et al., 2020). Nagata et al. (2021) evidenciaram um aumento na insegurança alimentar em populações vulneráveis durante a pandemia nos Estados Unidos. O estudo considerou o parâmetro de insuficiência alimentar que aumentou de $8,1 \%$ para $10,0 \%$ no período de março a junho de 2020, estando associado à perda recente de emprego, bem como redução da renda familiar, entre outros parâmetros socioeconômicos. $\mathrm{O}$ aumento da insegurança alimentar também foi observado na Índia por Hamadani et al. (2020), que verificaram redução da renda familiar da maioria dos participantes da pesquisa e, consequentemente o aumento da insegurança alimentar, que já era frequente no país.

Nicoletti et al. (2020) verificaram ingestão nutricional inadequada durante a quarentena de COVID-19 em pacientes submetidos à cirurgia bariátrica, caracterizada por consumo insuficiente de proteínas e uma ingestão relativamente alta de alimentos ultraprocessados. Segundo a Sbcbm (2020), os pacientes bariátricos devem manter o acompanhamento médico, nutricional e psicológico, a prática regular de atividade física, bem como seguir as recomendações nutricionais. Os resultados verificados por Nicoletti et al. (2020) demonstram o quanto a pandemia pode ter afetado o manejo de doenças crônicas e de condições de saúde em que o acompanhamento multiprofissional, estilo de vida e a alimentação compõem o tratamento.

\section{Conclusão}

A pandemia da COVID-19 alterou a rotina dos brasileiros, gerando impactos diretos nos seus hábitos alimentares, bem como em seu estilo de vida. A maioria dos estudos avaliados revelou alterações negativas nos hábitos alimentares dos 
brasileiros, que passaram a consumir mais alimentos ultraprocessados durante a pandemia, representando um comportamento de risco para o desenvolvimento de DCNT.

Destaca-se a implicação da pandemia em aspectos relacionados à saúde mental, à alimentação emocional, bem como aspectos socioeconômicos, como redução da renda familiar. Tais aspectos podem estar diretamente relacionados ao aumento no consumo de alimentos não saudáveis durante a pandemia da COVID-19.

Alguns estudos indicaram manutenção ou aumento no consumo de alimentos saudáveis, sugerindo que há uma preocupação com a ingestão de alimentos saudáveis que auxiliem no bom funcionamento do organismo e na melhora na imunidade. Outra razão para esse aumento pode estar associada ao maior tempo de permanência em casa, o que pode ter resultado em maior frequência de refeições preparadas e consumidas no domicílio.

Por fim, destaca-se que pesquisas sobre essa temática são incipientes no Brasil, sendo necessária a realização de estudos longitudinais que avaliem o impacto da pandemia da COVID-19 nos hábitos alimentares dos brasileiros e sua repercussão na saúde, qualidade de vida e segurança alimentar e nutricional a longo prazo. Estudos assim, podem embasar ações de enfrentamento à doença e aos desdobramentos desse fenômeno mundial na saúde do brasileiro.

\section{Referências}

Acter, T., Uddin, N., Das, J., Akhter, A., Choudhury, T. R., \& Kim, S. (2020). Evolution of severe acute respiratory syndrome coronavirus 2 (SARS-CoV-2) as coronavirus disease 2019 (COVID-19) pandemic: A global health emergency. The Science of the total environment, $730,138996$.

Bielemann, R. M, Motta, J. V. S, Minten, G. C, Horta, B. L, \& Gigante, D. P. (2015). Consumo de alimentos ultraprocessados e impacto na dieta de adultos jovens. Revista de Saúde Pública, 49: 28.

Brasil. Ministério da Saúde. Painel de casos de doença pelo coronavírus 2019 (COVID-19) no Brasil [Internet]. Brasília: Ministério da Saúde. (2020). https://covid.saude.gov.br/

Campagnaro, R., Collet, G. O., Andrade, M. P., Salles, J., Calvo Fracasso, M. L., Scheffel, D., Freitas, K., \& Santin, G. C. (2020). COVID-19 pandemic and pediatric dentistry: Fear, eating habits and parent's oral health perceptions. Children and youth services review, $118,105469$.

Cecchetto, C., Aiello, M., Gentili, C., Ionta, S., \& Osimo, S. A. (2021). Increased emotional eating during COVID-19 associated with lockdown, psychological and social distress. Appetite, 160, 105122.

da Mota Santana, J., Milagres, M. P., Dos Santos, C. S., Brazil, J. M., Lima, E. R., \& Pereira, M. (2021). Dietary intake of university students during COVID19 social distancing in the Northeast of Brazil and associated factors. Appetite, 105172.

Dias, A. D. C., de Sousa Araújo, D. G., da Silva, E. M., Farias, I. M., \& de Freitas Gomes, L. M. (2020). A importância da alimentação saudável e estado nutricional adequado frente a pandemia de COVID-19. Brazilian Journal of Development, 6(9), 66464-66473.

GBD 2017 Causes of Death Collaborators (2018). Global, regional, and national age-sex-specific mortality for 282 causes of death in 195 countries and territories, 1980-2017: a systematic analysis for the Global Burden of Disease Study 2017. Lancet (London, England), 392(10159), $1736-1788$.

Gomes, G.S. (2020). Aprendizagem em dias de pandemia: das lições do home office e alternativas para a gestão e disseminação do conhecimento. Revista Jurídica do Ministério Público do Estado do Tocantins. 1(18).

Hamadani, J. D., Hasan, M. I., Baldi, A. J., Hossain, S. J., Shiraji, S., Bhuiyan, M., Mehrin, S. F., Fisher, J., Tofail, F., Tipu, S., Grantham-McGregor, S., Biggs, B. A., Braat, S., \& Pasricha, S. R. (2020). Immediate impact of stay-at-home orders to control COVID-19 transmission on socioeconomic conditions, food insecurity, mental health, and intimate partner violence in Bangladeshi women and their families: an interrupted time series. The Lancet. Global health, $8(11)$, e1380-e1389.

Haushofer, J., \& Metcalf, C. (2020). Which interventions work best in a pandemic? Science, 368(6495), $1063-1065$.

Jones, B. L. (2018). Making time for family meals: Parental influences, home eating environments, barriers and protective factors. Physiology \& behavior, $193,248-251$

Klering, M. A. V., \& Neto, J. A. R. G. (2020). Flexibilização das leis trabalhistas frente à crise econômica amplificada pela pandemia do covid-19. Anuário Pesquisa e Extensão Unoesc São Miguel do Oeste. 5, e24538-e24538.

López-Moreno, M., López, M., Miguel, M., \& Garcés-Rimón, M. (2020). Physical and Psychological Effects Related to Food Habits and Lifestyle Changes Derived from Covid-19 Home Confinement in the Spanish Population. Nutrients, 12(11), 3445.

Lu, R., Zhao, X., Li, J., Niu, P., Yang, B., Wu, H., Wang, W., Song, H., Huang, B., Zhu, N., Bi, Y., Ma, X., Zhan, F., Wang, L., Hu, T., Zhou, H., Hu, Z., Zhou, W., Zhao, L., Chen, J., \& Tan, W. (2020). Genomic characterisation and epidemiology of 2019 novel coronavirus: implications for virus origins and receptor binding. Lancet (London, England), 395(10224), 565-574.

Malta, D. C., Szwarcwald, C. L., Barros, M. B. D. A., Gomes, C. S., Machado, Í. E., \& Júnior, P. R. B. D. S., et al. (2020). A pandemia da COVID-19 e as mudanças no estilo de vida dos brasileiros adultos: um estudo transversal, 2020. Epidemiologia e Serviços de Saúde, 29 (4), e2020407. 
Mantau, A., Hattula, S., \& Bornemann, T. (2018). Individual determinants of emotional eating: A simultaneous investigation. Appetite, 130, 93-103.

Marrón-Ponce, J. A., Flores, M., Cediel, G., Monteiro, C. A., \& Batis, C. (2019). Associations between Consumption of Ultra-Processed Foods and Intake of Nutrients Related to Chronic Non-Communicable Diseases in Mexico. Journal of the Academy of Nutrition and Dietetics, 119(11), $1852-1865$.

Mattioli, A. V., Pinti, M., Farinetti, A., \& Nasi, M. (2020). Obesity risk during collective quarantine for the COVID-19 epidemic. Obesity medicine, 20, 100263.

McAtamney, K., Mantzios, M., Egan, H., \& Wallis, D. J. (2021). Emotional eating during COVID-19 in the United Kingdom: Exploring the roles of alexithymia and emotion dysregulation. Appetite, 161, 105120. Advance online publication.

Menegassi, B., Almeida, J. B. D., Olimpio, M. Y. M., Brunharo, M. S. M., \& Langa, F. R. A nova classificação de alimentos: teoria, prática e dificuldades. (2018). Ciência \& Saúde Coletiva, 23, 4165-4176.

Mills, S., White, M., Brown, H., Wrieden, W., Kwasnicka, D., Halligan, J., Robalino, S., \& Adams, J. (2017) Health and social determinants and outcomes of home cooking: A systematic review of observational studies. Appetite, $111116 \mathrm{e} 134$.

Morato, M. M., Bastos, S. B., Cajueiro, D. O., \& Normey-Rico, J. E. (2020). An optimal predictive control strategy for COVID-19 (SARS-CoV-2) social distancing policies in Brazil. Annual reviews in control, 50, 417-431.

Nagata, J. M., Ganson, K. T., Whittle, H. J., Chu, J., Harris, O. O., Tsai, A. C., \& Weiser, S. D. (2021). Food Insufficiency and Mental Health in the US During the COVID-19 Pandemic. American Journal of Preventive Medicine. 60, 4, 453-461.

Nicoletti, C.F., Esteves, G.P., Genario, R., Santo, M.A., De Cleva, R., Gualano, B., \& Roschel, H. (2020). Nutritional Inadequacies Among Post-bariatric Patients During COVID-19 Quarantine in Sao Paulo, Brazil. OBES SURG.

Pan, K. Y., Kok, A., Eikelenboom, M., Horsfall, M., Jörg, F., Luteijn, R. A., Rhebergen, D., Oppen, P. V., Giltay, E. J., \& Penninx, B. (2021). The mental health impact of the COVID-19 pandemic on people with and without depressive, anxiety, or obsessive-compulsive disorders: a longitudinal study of three Dutch case-control cohorts. The lancet. Psychiatry, 8(2), 121-129.

Penne, T., \& Goedeme, T. B. F. (2020). Can low-income households afford a healthy diet? Insufficient income as a driver of food insecurity in Europe. Food Policy.

Ribeiro-Silva, R. D. C., Pereira, M., Campello, T., Aragão, É., Guimarães, J. M. D. M., Ferreira, A. J., \& Santos, S. M. C. D. (2020). Implicações da pandemia COVID-19 para a segurança alimentar e nutricional no Brasil. Ciência \& Saúde Coletiva, 25, 3421-3430.

Rodriguez-Morales, A. J., Gallego, V., Escalera-Antezana, J. P., Méndez, C. A., Zambrano, L. I., Franco-Paredes, C., Suárez, J. A., Rodriguez-Enciso, H. D., Balbin-Ramon, G. J., Savio-Larriera, E., Risquez, A., \& Cimerman, S. (2020). COVID-19 in Latin America: The implications of the first confirmed case in Brazil. Travel medicine and infectious disease, $35,101613$.

Ruiz-Roso, M. B., de Carvalho Padilha, P., Mantilla-Escalante, D. C., Ulloa, N., Brun, P., Acevedo-Correa, D., Arantes Ferreira Peres, W., Martorell, M., Aires, M. T., de Oliveira Cardoso, L., Carrasco-Marín, F., Paternina-Sierra, K., Rodriguez-Meza, J. E., Montero, P. M., Bernabè, G., Pauletto, A., Taci, X., Visioli, F., \& Dávalos, A. (2020). Covid-19 Confinement and Changes of Adolescent's Dietary Trends in Italy, Spain, Chile, Colombia and Brazil. Nutrients, 12(6), 1807.

Sayeed, A., Kundu, S., Banna, M., Hasan, M. T., Begum, M. R., \& Khan, M. (2020). Mental health outcomes during the COVID-19 and perceptions towards the pandemic: Findings from a cross sectional study among Bangladeshi students. Children and youth services review, 119, 105658.

Sbcbm (2020). Recomendação da Sociedade Brasileira de Cirurgia Bariátrica e Metabólica (SBCBM) em razão do Coronavírus - COVID-19. https://www.sbcbm.org.br/recomendacao-da-sociedade-brasileira-de-cirurgia-bariatrica-e-metabolica-sbcbm-em-razao-do-coronavirus-covid/

Sosa, M. A. M., Valdés, R. A. Á., \& Molina, F. J. R. (2020). COVID-19: deadly disease for patients with chronic non-communicable diseases. Revista Científica Estudiantil de Cienfuegos INMEDSUR, 3(2), 64-70, 2020.

Souza, M. T., Silva, M. D., \& Carvalho, R. (2010). Revisão integrativa: o que é e como fazer. Einstein (São Paulo), 8, (1), 102-106.

Steele, E. M., Rauber, F., Costa, C. D. S., Leite, M. A., Gabe, K. T., Louzada, M. L. D. C., \& Monteiro, C. A. (2020). Mudanças dietéticas na coorte NutriNet Brasil durante a pandemia covid-19. Revista de Saúde Pública, 54, 91.

Suggs, L. S., Della Bella, S., Rangelov, N., \& Marques-Vidal, P. (2018). Is it better at home with my family? The effects of people and place on children's eating behavior. Appetite, 121, 111-118.

Torres, B. L. P. M., Pinto, S. R. R., Dos Santos Silva, B. L., Dos Santos, M. D. D. C., Moura, A. C. C., Luz, L. C. X., \& De Carvalho, C. M. R. G. (2020). Reflexões sobre fatores determinantes dos hábitos alimentares na infância. Brazilian Journal of Development, 6, (9), 66267-66277.

Veiga, N. H., Ten, Y. Z. L. F., Machado, V. P., De Araujo Faria, M. G., De Oliveira Neto, M., \& David, H. M. S. L. (2021). Teoria da adaptação e saúde do trabalhador em home office na pandemia de covid-19. Revista Baiana de Enfermagem, 35: e37636.

Werneck, A. O., Silva, D. R. D., Malta, D. C., Souza-Júnior, P. R. B. D., Azevedo, L. O., Barros, M. B. D. A., \& Szwarcwald, C. L. (2020). Lifestyle behaviors changes during the COVID-19 pandemic quarantine among 6,881 Brazilian adults with depression and 35,143 without depression. Ciência \& Saúde Coletiva, 25, 4151-4156.

Werneck, A. O., Silva, D. R., Malta, D. C., Gomes, C. S., Souza-Júnior, P. R., Azevedo, L. O., Barros, M. B., \& Szwarcwald, C. L. (2021). Associations of sedentary behaviours and incidence of unhealthy diet during the COVID-19 quarantine in Brazil. Public health nutrition, 24(3), 422-426.

World Health Organization (2020a). WHO Director-General's opening remarks at the media briefing on COVID-19 - 11 March 2020 . World Health 
Research, Society and Development, v. 10, n. 4, e29810414125, 2021

(CC BY 4.0) | ISSN 2525-3409 | DOI: http://dx.doi.org/10.33448/rsd-v10i4.14125

Organization. https://www.who.int/dg/speeches/detail/who-director-general-s-opening-remarks-at-the-media-briefing-on-covid-19---11-march-2020/

World Health Organization (2020b). Novel Coronavirus (2019-nCoV): situation report, $12 . \quad$ World Health Organization. https://apps.who.int/iris/handle/10665/330777

World Health Organization. Diet, nutrition and the prevention of chronic diseases: Report of a Joint WHO/FAO Expert Consultation. Geneva; 2003. (WHO Technical Report Series, 916).

Zarnowiecki, D. M., Dollman, J. \& Parletta, N. Associations between predictors of children's dietary intake and socioeconomic position: A systematic review of the literature. Obes. Rev. 2014, 15, 375-391. 